\section{SEVERITY OF ILLNESS}

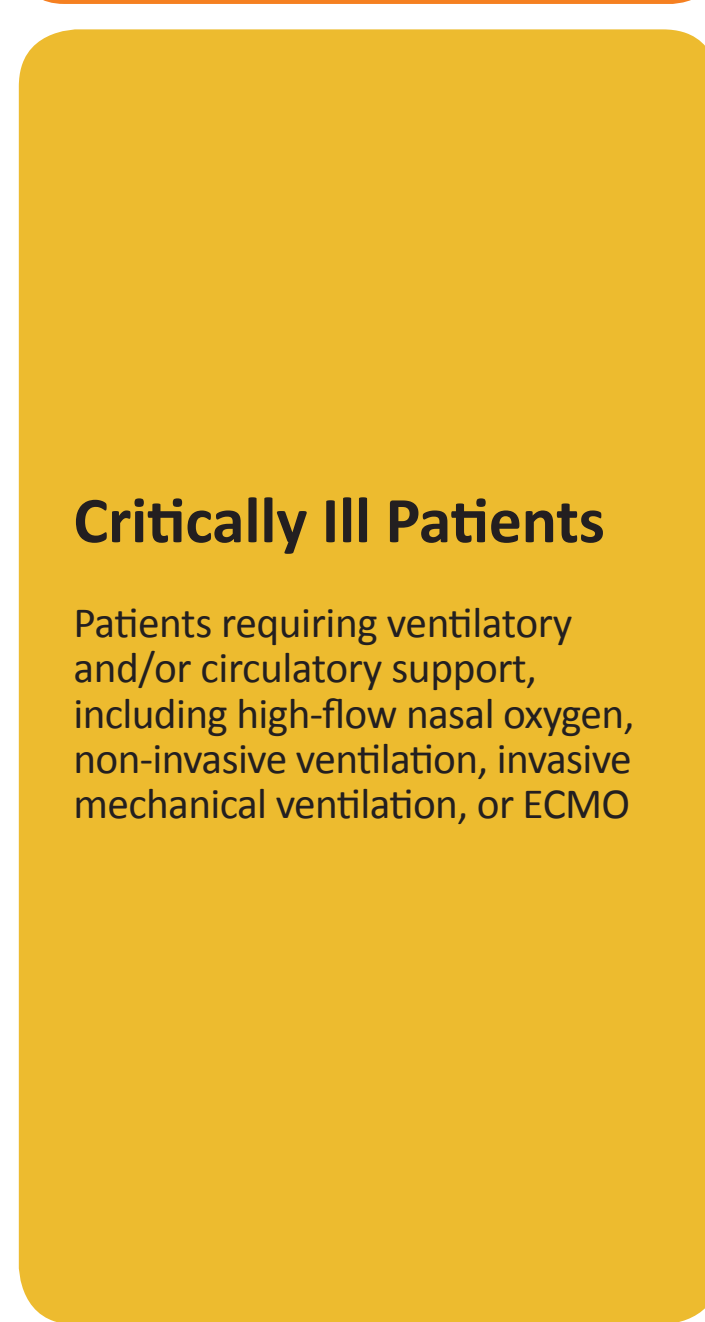

Moderately III Patients

Patients newly requiring low-flow supplemental oxygen

Mildly III Patients

\title{
RECOMMENDATIONS
}

Dexamethasone $6 \mathrm{mg} \mathrm{PO/IV} \mathrm{daily} \mathrm{for} 10$ days (or until discharge if sooner) is recommended.

Tocilizumab is recommended for patients who are on recommended doses of dexamethasone therapy (or a dose-equivalent corticosteroid) AND are within 14 days of .

\section{RECOMMENDATIONS FOR DRUG SHORTAGE SITUATIONS} In drug shortage situations, a single dose of tocilizumab $400 \mathrm{mg}$ IV or sarilumab
$400 \mathrm{mg}$ IV should be used for all eligible patients. A second dose of tocilizumab or sarilumab should notbe given to any patient.

Baricitinib $4 \mathrm{mg} \mathrm{PO} / \mathrm{NG}$ daily for 14 days (or until discharge if sooner) is recommended in patients who are on recommended doses of dexamethasone therapy (or a dose-equivalent corticosteroid) or who have a contraindication baricitinib and IL-6 inhibitors due to absence of safety and efficacy evidon

Dexamethasone $12 \mathrm{mg} \mathrm{PO} / \mathrm{IV}$ daily for 10 days (or until dischar worer) $\mathrm{ma}$ to receive IL-6 inhils (tocilizumab, sarilumab) or baricitinib. This recommendation is basen ver
evidence of reduction in days alive without life sun inpatient treatment options with a reasonablo vrofile durin spike in COVID-19 cases due
IL-6 inhibitors and baricitinib.

$\frac{\text { Dexan }}{\text { If } p a t i c}$
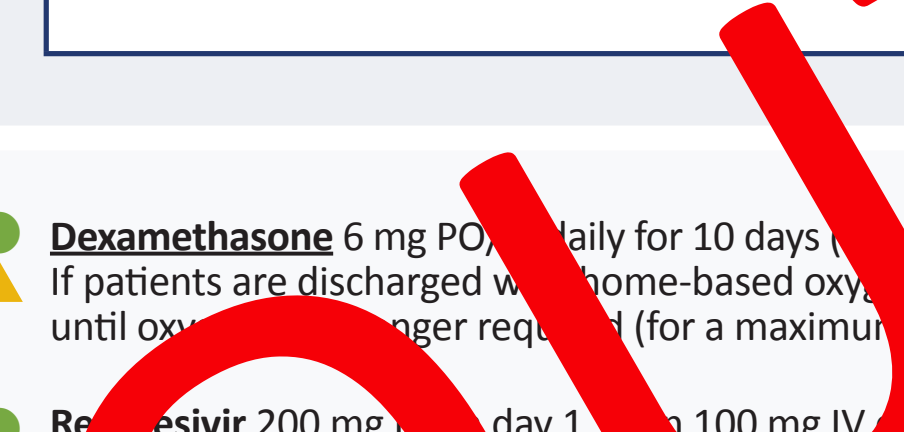

Re esivir $200 \mathrm{mg}$ day 1 ,

$\triangle$ I ppeutic dose antic

al agulation in patier
Al

isidered over prophylactic dose

SARS- neutrali- antibodies are not recommended for moderately ill patients. SARS-
For symp neutrali- antibodies are not recommended for moderately ill patients.
sotrovimab orr page 2 .

nirmatrelvir/ritonavir (Paxlovid) is not recommended for moderately ill patients.

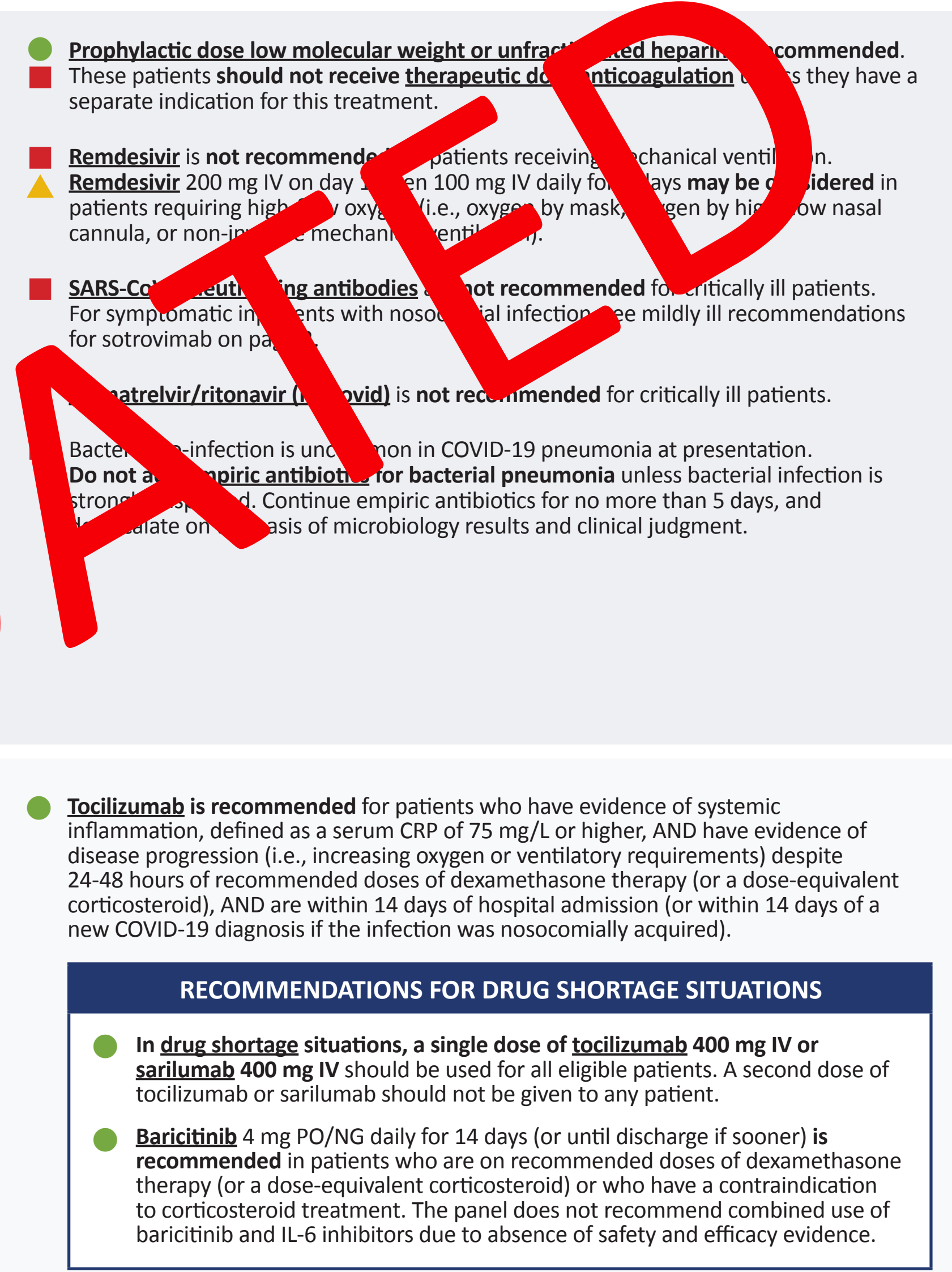

CURRENTLY NOT RECOMMENDED*

There is insufficient evidence to support the use of the
following therapies in the of clinical trials or where other indications would justify its use

$\checkmark$ Colchicine

$\checkmark$ Interferon (with or without lopinavir-ritonavir and

Vitamin D

RECOMMENDED AGAINST*

The following therapies are not
recommended for treatment of recommended for treatment of
COVID-19 due to lack of benefit potential harm, and system
mplications of overuse:

Antibiotics (azithromycin)

Casirivimab-imdevimab Casirivimab-imdevimab activity against the

Hydroxychloroquine or

chloroquine

Ivermectin

Lopinavir/ritonavir

* Applies to patients with any 


\section{Mildly III Patients}

Patients who do not require new or additional supplemental oxygen

\section{Tier 1}

Immunocompromised individuals not expected to mount an adequate immune response SARS-CoV-2 infection due to their underlying conditions, regardless of vaccine status; $O R$ Unvaccinated ${ }^{2}$ individuals at highest risk of severe disease Indigenous and age $>60$ years, or age $\geq 60$ years with one or more risk factors ${ }^{3}$ ). Older ire at higher risk prioritized for treatment in this tier. $^{4}$

\section{Tier 2}

Unvaccinated ${ }^{2}$ individuals at risk of severe disease (only if also age
$\geq 60$ years, Indigenous and age $\geq 50$ years, or $\geq 50$ years with one or

\section{Tier 3}

Vaccinated individuals at highest age $\geq 70$ years, Indigenous and age $\geq 60$ years, or age $\geq 60$ years
with one or more risk factors $\left.{ }^{3}\right)$. Vaccinated individuals who are
$>6$ months from their last dose $>6$ months from their last dos of vaccine are at higher risk,
and should be prioritized for treatment in this tier. ${ }^{4}$

\section{Tier 4}

Vaccinated individuals at risk of severe disease (only if also age $\geq 60$ years, Indigenous and age $\geq 50$ years, or $\geq 50$ years with one
or more risk factors ${ }^{3}$. Vaccinated individuals who are $>6$ months from their last dose of vaccine are at higher risk, and should be or treatment in this tier. $^{4}$

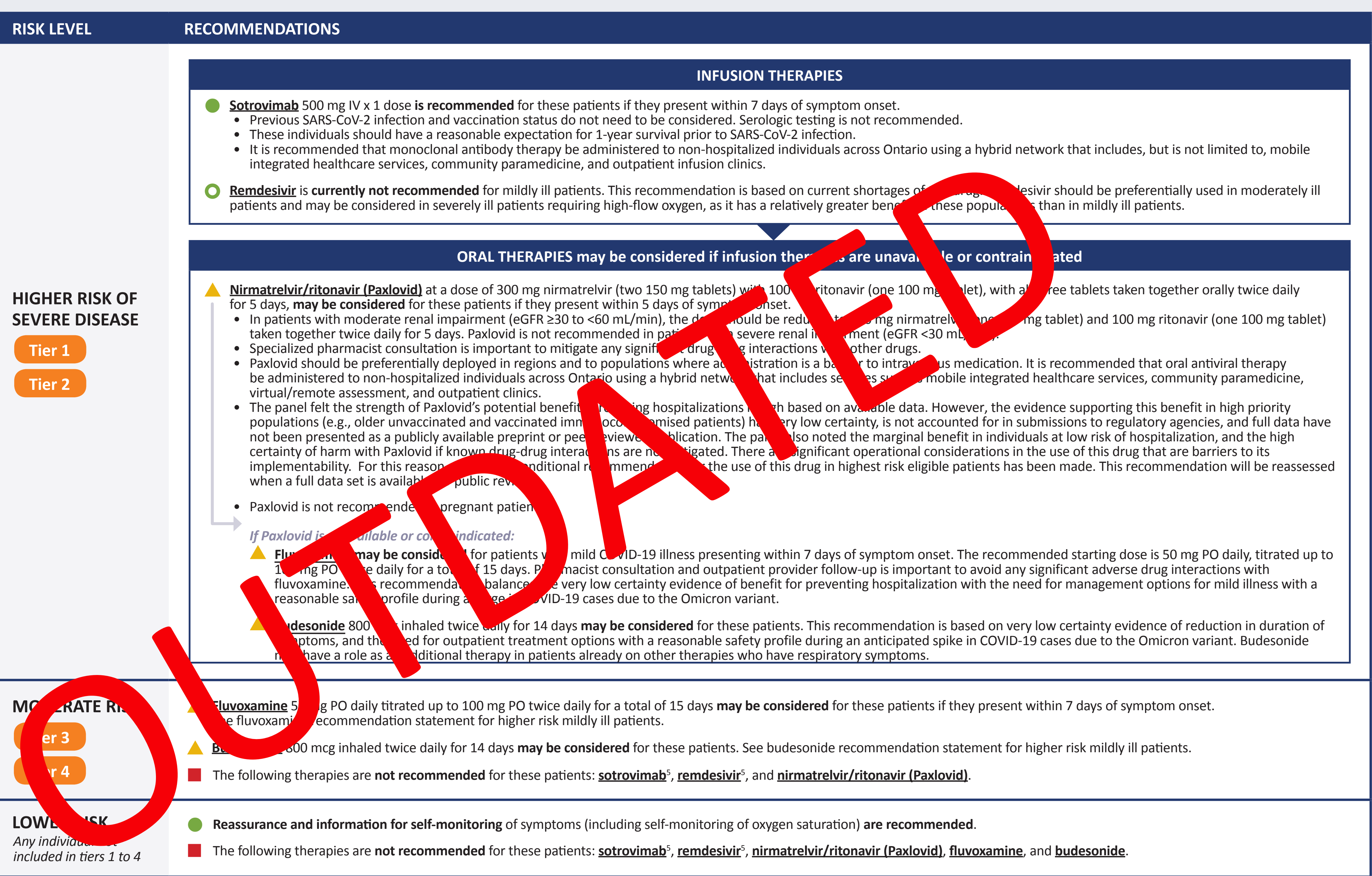

There is currently insufficient evidence to make a recommendation around aspirin or anticoagulation for mildly ill patients.

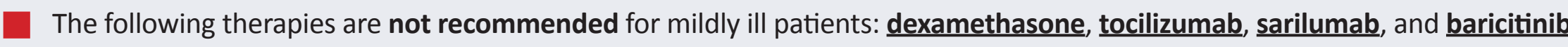

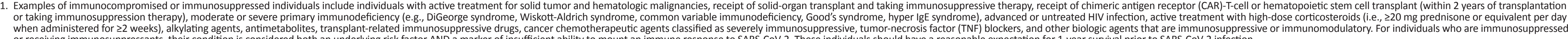

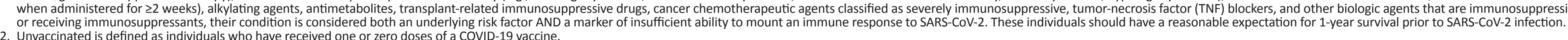

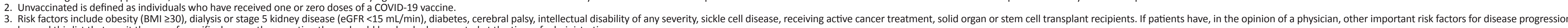

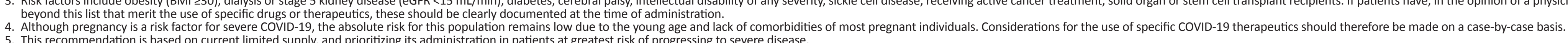

